\title{
Implicaciones clínicas e inmunológicas de los micronutrientes durante la infección por VIH
}

\author{
Clinical and immunological implications of micronutrients \\ during HIV infection \\ Implicações clínicas e imunológicas dos micronutrientes \\ durante a infecção pelo HIV
}

Iván Armando Osuna-Padilla' ${ }^{1}$, Nadia Carolina Rodríguez Moguel'1, Adriana Aguilar Vargas', Olivia Briceño ${ }^{*}$

Recibido: 26 de junio de 2020. Aceptado para publicación: 5 de agosto de 2020

Publicado en línea: 5 de septiembre de 2020

https://doi.org/10.35454/rncm.v3n2.166

\section{Resumen}

Durante la infección por VIH existe una alteración en las funciones del sistema inmunológico e inflamación crónica que no logra resolverse con el tratamiento antirretroviral (TAR). Aunado a lo anterior, se ha reportado una alta prevalencia de deficiencia de micronutrientes en la población $\mathrm{VIH}+$ debido a un aumento en la demanda y en la excreción de los mismos.

Dicha inflamación crónica y deficiencia de micronutrientes está asociada a la aparición de comorbilidades no asociadas al síndrome de inmunodeficiencia adquirida (SIDA) como hipertensión arterial, enfermedades cardiovasculares, síndrome metabólico, cáncer y osteoporosis. Es necesario desarrollar estrategias dirigidas a regular la inflamación y activación crónica presente en los individuos $\mathrm{VIH}+$ bajo TAR para mejorar su calidad de vida y disminuir la prevalencia de comorbilidades. Una opción prometedora es la intervención nutricional a través de la suplementación de micronutrientes que han demostrado tener un efecto regulador de la inflamación y de la respuesta inmunológica y que podrían representar una opción segura y costo eficiente.

En la presente revisión se explorarán las implicaciones clínicas e inmunológicas de los micronutrientes en individuos infectados con VIH a través del análisis de los es-
Abstract

During HIV infection there is an alteration in the functions of the immune system and chronic inflammation that cannot be resolved with antiretroviral treatment (ART). Moreover, a high prevalence of micronutrient deficiencies has been reported in the HIV+ population due to an increase in their demand and excretion.

Such chronic inflammation and micronutrient deficiency is associated with comorbidities not linked to the acquired immunodeficiency syndrome (AIDS) such as high blood pressure, cardiovascular disease, metabolic syndrome, cancer, and osteoporosis. The development of strategies aimed at regulating the inflammation and chronic activation present in HIV+ patients receiving ART to improve their quality of life and decrease the prevalence of comorbidities. A promising option is nutritional intervention through supplementation of micronutrients that have shown to have a regulatory effect on inflammation and immune response and which could represent a safe and cost-effective option.

In the current review, the clinical and immunological implications of micronutrients in HIV-infected individuals will be explored by reviewing clinical studies on supplementation in this population, and recommendations will be issued for the detection of individuals at risk of micronu-

\section{Resumo}

Durante a infeção pelo HIV, há uma alteração nas funções do sistema imunológico e uma inflamação crônica que não se podem resolver com o tratamento antirretroviral (TAR). Além disso, se tem registado uma alta prevalência de deficiência de micronutrientes na população HIV+ devido a um aumento no seu consumo e na sua excreção.

Essa inflamação crônica e deficiência de micronutrientes estão associadas ao aparecimento de comorbilidades não associadas à SIDA, como hipertensão arterial, doenças cardiovasculares, síndrome metabólico, câncer e osteoporose. É necessário desenvolver estratégias destinadas a regular a inflamação e a ativação crônica presentes em indivíduos HIV+ sob TAR para melhorar sua qualidade de vida e diminuir a prevalência de comorbilidades. Uma opção promissora é a intervenção nutricional através da suplementação de micronutrientes que demonstraram ter um efeito regulador na inflamação e na resposta imunológica e que poderiam representar uma opção segura e de baixo custo.

$\mathrm{Na}$ presente revisão, se exploram as implicações clínicas e imunológicas dos micronutrientes em indivíduos infetados com HIV e através da análise dos estudos clínicos de suplementação nessa população, serão emitidas recomendações para a deteção de indivíduos em risco de defi- 
tudios clínicos de suplementación en esta población y se emitirán recomendaciones para la detección de individuos en riesgo de deficiencia de micronutrientes y su manejo nutricional.

trient deficiency and its nutritional management.

Keywords: Nutritional therapy; Micronutrients; HIV; Inflammation. ciência de micronutrientes e seu acompanhamento nutricional.

Palavras-chave: terapia nutricional, micronutrientes, HIV, inflamação.

Palabras clave: terapia nutricional, micronutrientes, VIH, inflamación.

Centro de Investigación en Enfermedades Infecciosas, Instituto Nacional de Enfermedades Respiratorias, CDMX, México.
*Correspondencia: Olivia Briceño olivia@cieni.org.mx

\section{INTRODUCCIÓN}

A pesar de que la infección por el virus de la inmunodeficiencia humana (VIH) ha sido extensamente estudiada durante casi 40 años, aún no ha sido posible obtener una cura o una vacuna. Sin embargo, el desarrollo de fármacos antivirales ha establecido un avance sin precedentes en el que los individuos bajo tratamiento antirretroviral (TAR) tienen un control de la carga viral a niveles indetectables a largo plazo, así como una esperanza de vida muy similar a la población sin infección por VIH. Hoy en día, el reto que enfrenta el personal de salud para el manejo de los individuos infectados con VIH bajo TAR exitoso es la alta prevalencia de comorbilidades no asociadas al síndrome de inmunodeficiencia adquirida (SIDA) tales como hipertensión arterial, enfermedades cardiovasculares, síndrome metabólico, cáncer y osteoporosis, entre otras ${ }^{(1)}$. La aparición de dichas comorbilidades ha sido asociada a la presencia de inflamación y activación crónica residual que no logra ser controlada con el TAR, así como con la deficiencia de micronutrientes clave. En este momento, para la comunidad científica es primordial estudiar los mecanismos involucrados en la persistencia y regulación de la inflamación y activación crónica presente en los individuos VIH+ con TAR. Una opción poco explorada, pero que representa un gran potencial, es la intervención nutricional a través de la suplementación con micronutrientes con efecto regulador de la respuesta inflamatoria y de modulación del sistema inmune, lo cual podría representar una opción segura y de bajo costo para mejorar la calidad de vida de esta población. En la presente revisión se explorarán las implicaciones clínicas e inmunológicas de los micronutrientes en individuos infectados con VIH a través del análisis de los estudios clínicos de su suplementación en esta población y se emitirán recomendaciones para la detección de individuos en riesgo de deficiencia de micronutrientes y su manejo nutricional.

Para la elaboración de este estudio, se realizó una búsqueda no sistemática de la literatura en las bases de datos Pubmed, Scielo y Cochrane Library para identificar estudios cuyo objetivo primario fuera la evaluación del efecto de la suplementación de micronutrientes en parámetros clínicos e inmunológicos realizados en individuos infectados con VIH. Se utilizaron las palabras clave micronutrient OR omega-3 OR vitamin D OR zinc OR selenium OR vitamin $A N D$ hiv $O R$ aids. Se incluyeron ensayos clínicos, revisiones sistemáticas y metaanálisis publicados en inglés, de enero de 1995 a junio de 2020.

\section{GENERALIDADES DEL VIH}

El VIH es un retrovirus con un genoma de $10 \mathrm{~kb}$ de longitud codificado por 2 cadenas de $\mathrm{ARN}^{(2)}$, el cual infecta a las células T-CD4+ que sufren una destrucción progresiva y con ello, llevan al individuo infectado a un estado de inmunosupresión. Después de 5 - 10 años en ausencia de TAR, se presenta un incremento en la susceptibilidad a infecciones oportunistas que indican la progresión de la enfermedad a $\operatorname{SIDA}^{(2,3)}$.

\section{Comorbilidades en el paciente con VIH}

Los avances en el tratamiento del VIH han permitido disminuir de forma importante la mortalidad asociada al SIDA; sin embargo, la presencia de inflamación crónica y activación inmunológica persistente se han relacionado con la aparición de comorbilidades no asociadas al SIDA, entre las que destacan las alteraciones metabólicas, enfermedad cardiovascular, cáncer, osteoporosis y obesidad ${ }^{(4-6)}$. 
La aparición de comorbilidades en individuos infectados por VIH bajo TAR obedece a múltiples factores entre los que destacan el desgaste del sistema inmunológico tras una exposición crónica a niveles de inflamación elevados, la presencia de otras coinfecciones activas simultáneas a VIH tales como citomegalovirus o hepatitis y a efectos adversos asociados al uso prolongado de $\mathrm{TAR}^{(4)}$. Así mismo, se han descrito aspectos sociales tales como un estilo de vida poco saludable, la inseguridad social y alimentaria, y la deficiencia de micronutrientes como factores asociados a la alta prevalencia de comorbilidades en individuos infectados por $\mathrm{VIH}^{(5)}$.

\section{Comorbilidades metabólicas asociadas a la terapia antirretroviral}

Se ha documentado una gran incidencia de enfermedades crónicas no transmisibles en individuos VIH+ tratados. La prevalencia de dislipidemia oscila entre $74 \%$ en aquellos que reciben TAR y $26 \%$ en pacientes sin tratamiento ${ }^{(7)}$. Así mismo, la infección por VIH y el TAR ocasionan cambios en las concentraciones de lípidos séricos, disminuyendo el colesterol de alta densidad (HDL) e incrementando el colesterol de baja densidad (LDL), colesterol total y triglicéridos ${ }^{(8)}$.

La exposición crónica al TAR, principalmente a inhibidores nucleósidos de la transcriptasa reversa (INTR) e inhibidores de la proteasa (IP), ocasiona resistencia a la insulina al afectar el metabolismo del transportador de la glucosa (GLUT-4), además de disminuir la secreción de insulina ${ }^{(9)}$.

El aumento en marcadores de estrés oxidativo es otra de las complicaciones reportadas en individuos VIH+ tratados, mostrando incremento en las moléculas anión superóxido, malondialdehído (MDA), especies reactivas de oxígeno (ERO) y productos de oxidación proteica después de iniciar $\operatorname{TAR}^{(10)}$.

\section{Comorbilidades asociadas a la activación inmunológica}

La respuesta inmune de defensa inicial del huésped ante el VIH es orquestada por el sistema inmune innato constituido por granulocitos, natural killers, monocitos, macrófagos y células dendríticas que tienen función fagocítica y producen factores antivirales que tienen por objetivo contener y eliminar al virus de forma inmediata, así como atraer a las células del sistema inmune adaptativo para montar una respuesta de memoria. Para el establecimiento de esta respuesta adaptativa (linfocitos T y B) se requieren mediadores inflamatorios solubles, conocidos como citocinas, entre las que destacan el factor de necrosis tumoral (TNF- $\alpha$ ) y las interleucinas-1 (IL-1) y 6 (IL-6) ${ }^{(3)}$. Estas moléculas ejercen una función protectora en el control de infecciones, la reparación tisular y el restablecimiento de la homeostasis; sin embargo, su producción y exposición crónica durante la infección por VIH genera un estado de hiperactivación ${ }^{(11)}$.

Diversos estudios han mostrado que la infección por VIH está asociada a una mayor frecuencia de monocitos proinflamatorios que correlaciona directamente con la carga viral, la activación de linfocitos T-CD8+, disminución en el conteo de linfocitos T-CD4+ en sangre periférica y con los niveles circulantes de IL-6 ${ }^{(12,13)}$. Estas alteraciones se han relacionado con un mayor riesgo cardiovascular ${ }^{(14)}$, además de incrementar el riesgo de desarrollar alteraciones en la densidad mineral ósea (DMO) al actuar sobre vías de señalización relacionadas con la resorción ósea ${ }^{(15)}$.

El VIH se replica en el tejido linfoide asociado al intestino, estimándose que $80 \%$ de los linfocitos $\mathrm{T}$-CD4+ residentes son destruidos en las primeras tres semanas posteriores a la infección ${ }^{(16)}$. Como resultado de este proceso, se genera daño en la mucosa intestinal y permeabilidad en el epitelio intestinal contribuyendo con ello a la translocación de lipopolisacárido (LPS), proveniente de las bacterias del microbioma intestinal, y que contribuye a la activación del sistema inmunológico y a un estado proinflamatorio crónico ${ }^{(17)}$. La resistencia a la insulina observada en la población VIH+, además de asociarse al uso de TAR, está directamente relacionada con las concentraciones plasmáticas de LPS $^{(18)}$. La diarrea también es una complicación intestinal frecuente. Cerca de $40 \%$ de los pacientes que viven con el VIH reportan al menos un episodio de diarrea al mes, mientras que $25 \%$ presentan diarrea crónica, viéndose incrementado el riesgo a desarrollar desnutrición como consecuencia de la malabsorción y las pérdidas aumentadas de macro y micronutrientes ${ }^{(19)}$.

Se ha observado que los altos niveles de activación y la respuesta alterada del sistema inmune persisten a pesar de estar bajo TAR por años ${ }^{(4)}$. Esto plantea un gran reto a la comunidad científica, ya que es necesario investigar nuevas estrategias para bloquear las fuentes de activación inmunológica e inflamación que están ocasionando un desgaste del sistema inmunológico, lo que conlleva a la aparición de comorbilidades y finalmente a la muerte de los individuos infectados con el VIH. 


\section{SUPLEMENTACIÓN DE MICRONUTRIENTES COMO COADYUVANTES DE LA TERAPIA ANTIRRETROVIRAL}

El estado inflamatorio crónico, la activación inmune residual y la presencia de estrés oxidativo condicionan un incremento en los requerimientos corporales de micronutrientes con actividad antioxidante e inmunomoduladora en los individuos VIH+. Esta situación se ve exacerbada ante un consumo subóptimo de los mismos, la presencia de agentes quelantes en la dieta como hierro y fibra, o bien por pérdidas excesivas de dichos nutrientes en casos de diarrea crónica ${ }^{(20)}$ (Figura 1). De ahí que diversos autores han evaluado el efecto clínico e inmunológico de la suplementación de diversos nutrientes en pacientes con el VIH, usando diversos desenlaces clínicos para medir su efectividad.

\section{Zinc}

El zinc es un elemento traza esencial en los humanos al ser un componente de una gran cantidad de enzimas y proteínas que participan en la regulación del sistema inmunológico ${ }^{(21)}$. En individuos sin infección por VIH, la deficiencia sérica se asocia a una disminución en la capacidad de proliferación, maduración y funcionalidad de distintas células del sistema inmune. Los macrófagos ven reducida su capacidad fagocítica, mientras que los linfocitos B disminuyen su nivel de maduración y su función de producción de inmunoglobulinas (anticuerpos) ${ }^{(22)}$. Así mismo, en individuos VIH+, en estados de deficiencia se reporta un incremento en las concentraciones de citocinas proinflamatorias y marcadores de estrés oxidativo, lo que contribuye al estado inflamatorio crónico ${ }^{(23,24)}$.
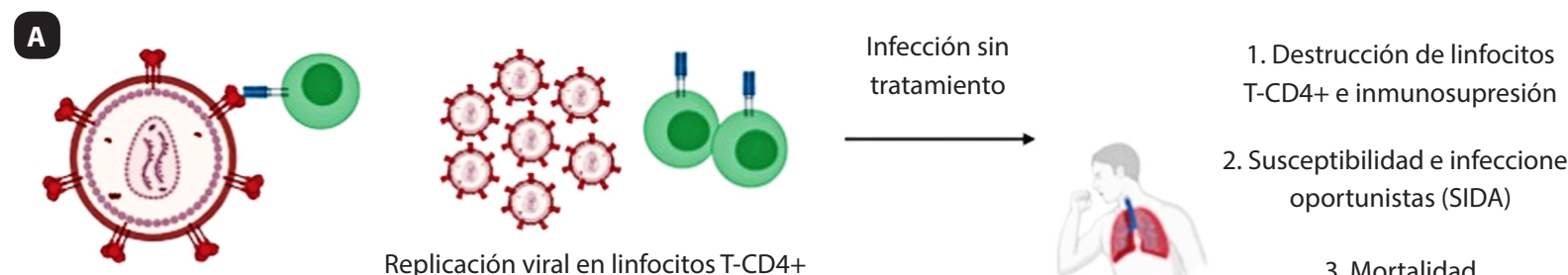

1. Destrucción de linfocitos T-CD4+ e inmunosupresión

2. Susceptibilidad e infecciones oportunistas (SIDA)

Replicación viral en linfocitos T-CD4+

3. Mortalidad

B
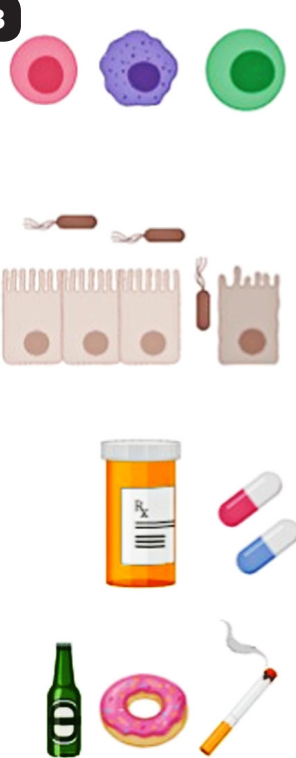

Activación inmunológica e incremento en la producción de citocinas proinflamatorias

(TNFa. IL-1, IL-6)

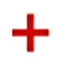

Pérdida de la integridad de la barrera intestinal, translocación bacteriana e inflamación sistémica

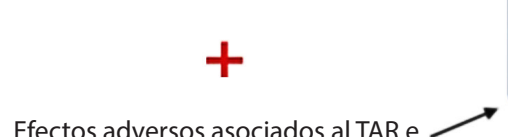
inflamación crónica de bajo grado

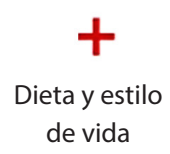

Deficiencia de micronutrientes

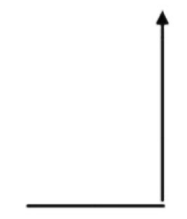

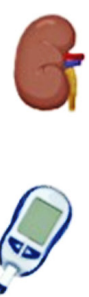
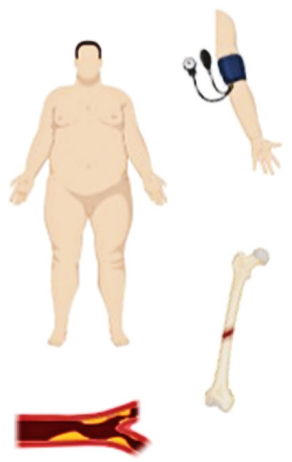

Comorbilidades no asociadas al VIH

Figura 1. Etiología de la deficiencia de micronutrientes en el paciente con el VIH. A. La infección por el VIH en ausencia de tratamiento antirretroviral (TAR) lleva a estados de inmunosupresión, mayor susceptibilidad a infecciones oportunistas, progresión de la enfermedad a SIDA y muerte. B. La activación del sistema inmune en respuesta a la infección por el VIH, la translocación de productos bacterianos del intestino, los efectos adversos asociados al TAR y un estilo de vida poco saludable condicionan un incremento en los requerimientos corporales de micronutrientes con actividad antioxidante e inmunomoduladora. Todos los factores anteriores están asociados a la aparición de comorbilidades no-asociadas a SIDA como la hipertensión, enfermedad cardiovascular, cáncer, obesidad y osteoporosis en los individuos VIH+. Diseñada por los autores. 
La prevalencia de deficiencia sérica de zinc en pacientes con el VIH es elevada. Martínez et al. reportaron en población estadounidense una prevalencia de deficiencia de $70,1 \%$, la cual es mayor $(82,3 \%)$ en pacientes coinfectados con hepatitis $C^{(23)}$. Nuestro grupo de investigación reportó una prevalencia de deficiencia de zinc de $23,9 \%$ en población mexicana $\mathrm{VIH}+$ bajo $\operatorname{TAR}^{(25)}$.

Considerando las altas tasas de deficiencia en la población VIH+, diversos autores han evaluado el impacto clínico e inmunológico de la suplementación de zinc en esta población. Mocchengiani et al. documentaron un incremento en las concentraciones séricas de zinc y en las cuentas de linfocitos T-CD4+ tras suplementar durante un mes una dosis de $200 \mathrm{mg}$ de sulfato de zinc $^{(26)}$. Bobat et al. evaluaron el efecto de la suplementación de $10 \mathrm{mg}$ de sulfato de zinc durante 6 meses en una muestra de niños $\mathrm{VIH}+$, documentando menor incidencia de episodios de diarrea ${ }^{(27)}$. Baum et al. suplementaron durante 18 meses $12 \mathrm{mg}$ de zinc en mujeres y $15 \mathrm{mg}$ en hombres de una población sin TAR, reportando disminución en falla inmunológica (lenta o nula recuperación de linfocitos T-CD4+ en sangre periférica) y menor incidencia de episodios de diarrea ${ }^{(28)}$.

Otros autores no han observado cambios tras suplementar distintas dosis de zinc. Green et al. suplementaron $50 \mathrm{mg}$ de sulfato de zinc durante 28 días a una población de pacientes $\mathrm{VIH}+$ con $\mathrm{TAR}^{(29)}$. Cárcamo et al. suplementaron en pacientes VIH+ con diarrea una dosis de $100 \mathrm{mg}$ de sulfato de zinc durante 14 días $^{(30)}$, mientras que Fawzi et al. y Villamor et al. evaluaron el efecto de la suplementación de $25 \mathrm{mg}$ de sulfato de zinc en mujeres embarazadas $\mathrm{VIH}+$, sin observar beneficios de la suplementación ${ }^{(31,32)}$.

A la fecha, no existe consenso de las dosis y tiempo a suplementar en pacientes con el VIH. Tampoco hay evidencia contundente sobre si las mediciones en sangre periférica o si las cantidades ingeridas son las más adecuadas para diagnosticar deficiencia de zinc. Sin embargo, los resultados de los estudios mostrados sugieren que la suplementación con zinc podría representar una alternativa para mejorar la respuesta inmunológica así como disminuir los episodios de diarrea, por lo que debe valorarse la suplementación a dosis menores a la ingesta máxima tolerada $(40 \mathrm{mg} /$ día considerando las recomendaciones emitidas por el Institute of Medicine de Estados Unidos) $)^{(33)}$, en individuos VIH+ con TAR en control virológico que no hayan mostrado reconstitución inmunológica o persistan con cuentas bajas de linfocitos T-CD4+ en sangre periférica, en individuos con diarrea crónica, en quienes se identifica un consumo subóptimo a través de herramientas para la evaluación dietética o bien en aquellos con signos clínicos de deficiencia.

\section{Selenio}

El selenio es un elemento traza requerido para la síntesis de selenocisteína y selenoproteínas, las cuales ejercen un papel importante en diferentes procesos celulares relacionados con la respuesta inmunológica, la regulación del estrés oxidativo y la inflamación ${ }^{(34)}$. Las células del sistema inmune expresan distintos tipos de selenoproteínas que participan en los procesos de activación, diferenciación y proliferación que permiten el establecimiento de la respuesta inmunológica en condiciones fisiológicas. Adicionalmente, el selenio es considerado un elemento antioxidante debido a su participación en la detoxificación del peróxido de hidrógeno o en la reversión de los efectos de la peroxidación lipídica ${ }^{(35)}$.

La prevalencia de deficiencia sérica de selenio durante la infección por VIH es alta, oscilando entre $53 \%$ $96 \%$, siendo mayor en pacientes $\sin \operatorname{TAR}^{(36,37)}$. En población mexicana, la prevalencia de deficiencia sérica de selenio en pacientes con TAR documentada es $65,9 \%{ }^{(25)}$. Algunas complicaciones propias de la infección por $\mathrm{VIH}$, como la diarrea, pueden exacerbar las deficiencias, reportándose concentraciones disminuidas de selenio hasta en $96 \%$ de los pacientes con VIH y diarrea, según el estudio realizado por Amare et al. ${ }^{(38)}$.

Diversos autores han evaluado el efecto de la suplementación de selenio en pacientes VIH+. Hurwitz et al. evaluaron el efecto de la suplementación de $200 \mu \mathrm{g} / \mathrm{d}$ de levadura de selenio durante 18 meses, documentando incremento en concentraciones séricas de selenio y en el conteo de linfocitos T-CD4+ en sangre periférica ${ }^{(39)}$. Kamwesiga et al. suplementaron durante dos años $200 \mu \mathrm{g} / \mathrm{d}$ de levadura de selenio en individuos sin TAR, reportando retraso en el declive de la cuenta de linfocitos T-CD4+ en comparación con individuos que recibieron placebo ${ }^{(40)}$. Hadadi et al. evaluaron el efecto de la suplementación de $200 \mu \mathrm{g} / \mathrm{d}$ de selenio en conjunto con $50 \mathrm{mg}$ de zinc elemental, no observando cambios en el conteo de células T-CD4+ en sangre periférica $^{(41)}$.

La mayoría de los resultados de los ensayos clínicos de suplementación de selenio en individuos VIH+ publicados a la fecha son poco concluyentes al tener como limitación principal un tamaño de muestra reducido, seguimientos clínicos con poca periodicidad o 
bien la ausencia de evaluación del consumo dietético de selenio en los participantes. Hasta el momento no ha sido evaluado el papel de la suplementación de selenio sobre la activación inmunológica, inflamación y estrés oxidativo en individuos VIH+ tratados. Si bien la deficiencia sérica de selenio reportada en esta población es elevada, no hay evidencia de que la suplementación mejore las concentraciones séricas, por lo que la intervención debe enfocarse en orientar al paciente a incrementar el consumo de las fuentes naturales de este nutriente a través de la dieta habitual, cubriendo la ingesta diaria recomendada para población sana emitida por el Institute of Medicine de Estados Unidos, la cual corresponde a $55 \mu \mathrm{g} /$ día para población mayor de 19 - 50 años y $45 \mu \mathrm{g} /$ día en mayores de 50 años ${ }^{(42)}$.

\section{Vitamina D}

El $80 \%$ - $90 \%$ de las concentraciones de esta vitamina liposoluble proviene de la exposición solar y la conversión a colecalciferol a nivel cutáneo, mientras que $10 \%$ a $20 \%$ dependen del consumo dietético ${ }^{(43)}$. La vitamina $\mathrm{D}$ en individuos sin infección por el VIH ha demostrado efectos en la inmunidad innata al incrementar la transcripción de péptidos antimicrobianos y la actividad fagocítica en monocitos - macrófagos, mientras que en la inmunidad adaptativa, se ha descrito un rol en la regulación de la producción de citocinas proinflamatorias, limitando la activación y la exacerbación de la respuesta inmune de las células $\mathrm{T}^{(44)}$. Con respecto a su papel en el metabolismo, las concentraciones óptimas de vitamina $\mathrm{D}$ se han relacionado con el adecuado funcionamiento de las células $\beta$-pancreáticas y la sensibilidad a la insulina, proponiéndose que a mayores concentraciones podría verse favorecida la síntesis y secreción de insulina ${ }^{(43)}$.

El estado proinflamatorio durante la infección por el VIH y la utilización de inhibidores no nucleósidos de la transcriptasa reversa (INNTR) e IP dentro del esquema de TAR ocasionan alteraciones en la funcionalidad de las hidroxilasas, disminuyendo la síntesis de la forma activa de la vitamina $\mathrm{D}^{(45)}$. La prevalencia de deficiencia de vitamina $\mathrm{D}$ en población VIH+ varía entre $25 \%$ y $78 \%{ }^{(46)}$, la cual se ha asociado a menores conteos de linfocitos T-CD4+ en sangre periférica e incremento en la producción de citocinas proinflamatorias y marcadores de activación inmune ${ }^{(47)}$. A nivel metabólico, la deficiencia de vitamina $\mathrm{D}$ en individuos $\mathrm{VIH}+$ se asocia a mayor riesgo de ateroesclerosis y enfermedades cardiovasculares ${ }^{(48)}$.
La asociación entre concentraciones disminuidas de vitamina $\mathrm{D}$ y el desarrollo de comorbilidades no asociadas a SIDA persiste aún en quienes reciben TAR ${ }^{(45)}$, por lo que se ha sugerido un potencial beneficio de su suplementación.

Eckard et al. evaluaron el efecto de tres dosis de vitamina D3 (18,000 - 60,000 - 120,000 UI/mes) durante 1 año en pacientes de 8 - 25 años infectados con el VIH en TAR con deficiencia sérica de vitamina $\mathrm{D}(<30 \mathrm{ng} /$ $\mathrm{mL}$ ), observando disminución en marcadores de activación de células T-CD4+, T-CD8+, monocitos proinflamatorios y marcadores de recambio óseo en el grupo que recibió $120,000 \mathrm{UI} / \mathrm{mes}^{(49,50)}$. Havens et al. documentaron beneficios en DMO lumbar tras suplementar 50.000 UI / día de vitamina D durante 48 semanas, en conjunto con un multivitamínico que contenía 400 UI de vitamina D3 y $162 \mathrm{mg}$ de calcio, esto en jóvenes de 16 - 24 años con el VIH en $\operatorname{TAR}^{(51)}$. Yin et al. evaluaron el impacto de la suplementación de 1,000 o 3,000 UI de vitamina D3 en mujeres posmenopáusicas infectadas con el VIH, documentando incremento en las concentraciones séricas de 25-OHD en el grupo que recibió 3,000 UI, sin efectos en la DMO tras 12 meses de suplementación ${ }^{(52)}$. Si bien algunos estudios han mostrado un efecto benéfico en la activación inmunológica tras la suplementación, los resultados con respecto a su impacto en la DMO son contradictorios debido a la heterogeneidad de las cohortes, por lo que aún no es posible dar recomendaciones claras para su suplementación en individuos VIH+. En ausencia de resultados significativos, la suplementación de vitamina $\mathrm{D}$ debe prescribirse únicamente en individuos $\mathrm{VIH}+$ con deficiencia sérica, promoviendo el consumo de alimentos ricos en vitamina $\mathrm{D}$ para asegurar la ingesta diaria recomendada (600 UI/día en menores de 70 años, 800 UI/ día en mayores de 70 años acorde con la recomendación del Institute of Medicine de Estados Unidos) ${ }^{(53)}$.

\section{Omega 3}

Los ácidos grasos omega 3 y 6 provenientes de la alimentación son incorporados a las membranas celulares, además de ser sustratos para la producción de eicosanoides (prostaglandinas, tromboxanos y leucotrienos) que participan como mediadores de la respuesta inflamatoria del sistema inmune. En general, los eicosanoides derivados de ácido araquidónico (omega 6) actúan en vías proinflamatorias, mientras que los derivados del ácido eicosapentaenoico (EPA) y ácido 
docosahexaenoico (DHA) se han asociado a menor potencial proinflamatorio y a la producción de mediadores pro-resolución (lipoxinas y resolvinas), inhibiendo así respuestas inflamatorias excesivas que se han relacionado con el desarrollo de enfermedades autoinmunes, inflamación crónica y daño tisular. Entre otros posibles beneficios asociados al omega 3 se encuentran el mantenimiento de la fluidez de la membrana celular, disminución en las concentraciones de citocinas proinflamatorias, mantenimiento de la integridad de la barrera intestinal y disminución en la síntesis de triglicéridos a nivel hepático en individuos sin infección por $\mathrm{VIH}^{(54)}$. Considerando el rol de estos ácidos grasos en la respuesta inmunológica y en el metabolismo en individuos sanos, diversos autores han evaluado el efecto de la suplementación en diferentes dosis en pacientes VIH+.

Metkus et al. suplementaron 3,6 g de omega 3 (645 mg de EPA y 365 mg de DHA) durante 8 semanas a pacientes VIH+ en control virológico que cursaban con hipertrigliceridemia, observando una disminución significativa en las concentraciones de IL-6 y TNF- $\alpha^{(55)}$. Dichos resultados fueron similares a lo reportado por Coghill et al. quienes suplementaron $3 \mathrm{~g}$ de omega 3 (1.800 mg de EPA y $1.200 \mathrm{mg}$ de DHA) durante 12 semanas a pacientes en fase de SIDA coinfectados con herpesvirus- $8^{(56)}$. Amador-Licona et al. evaluaron el impacto de los ácidos grasos en el estrés oxidativo, para lo cual suplementaron $2,4 \mathrm{~g}$ de omega 3 durante 6 meses en pacientes VIH+ bajo TAR, no observando una disminución en los marcadores de estrés oxidativo (glutatión, catabolitos de óxido nítrico y MDA) ${ }^{(57)}$. Se ha estudiado también el efecto de la suplementación de omega 3 en la modulación de la permeabilidad intestinal en individuos VIH+; tras suplementar 1,6 gramos de omega 3 ( $800 \mathrm{mg}$ de EPA y $600 \mathrm{mg}$ DHA) por 12 semanas, Zhang et al. observaron disminución en marcadores de inflamación y translocación bacteriana (CD14, citocinas proinflamatorias, LPS y proteína ligadora de LPS), lo que podría indicar una mejoría en la permeabilidad intestinal tras la suplementación ${ }^{(58)}$.

Otros autores han evaluado el efecto de la suplementación de aceite de pescado en el perfil de lípidos. Peters et al. evaluaron el efecto de $4 \mathrm{~g}$ de aceite de pescado (460 mg de EPA y $380 \mathrm{mg}$ de DHA) durante 12 semanas en pacientes VIH+ en TAR con diagnóstico de hipertrigliceridemia, documentando una disminución significativa en las concentraciones de triglicéridos al prescribirse de forma simultánea a fibratos ${ }^{(59)}$. Oliveira et al. evaluaron en una población brasileña en TAR el efecto de $3 \mathrm{~g}$ de aceite de pescado (540 mg de EPA y $360 \mathrm{mg}$ de DHA) en el perfil de lípidos, sin observar cambios significativos tras la suplementación ${ }^{(60)}$. Recientemente, Domingo et al. evaluaron el efecto clínico e inmunológico de la suplementación de 4.000 mg de DHA durante 48 semanas a pacientes con TAR, documentando una disminución significativa de triglicéridos y en la expresión de genes proinflamatorios en tejido adiposo subcutáneo ${ }^{(61)}$, reportando también un incremento en el colesterol LDL en el primer mes de suplementación, sin cambios en las concentraciones de colesterol HDL, insulina e índice de HOMA ${ }^{(62)}$.

Debido a la heterogeneidad de los resultados de los estudios reportados en la literatura, se han publicado dos metaanálisis de ensayos clínicos, cuyos resultados muestran una disminución significativa en las concentraciones de triglicéridos e incrementos en colesterol HDL tras la suplementación con ácidos grasos a individuos $\mathrm{VIH}+{ }^{(63,64)}$.

Considerando la falta de evidencia del efecto de la suplementación en el estado inflamatorio y estrés oxidativo, se debe considerar la suplementación de omega 3 (dosis de 2 - $3 \mathrm{~g} /$ día) solamente en pacientes $\mathrm{VIH}+$ con hipertrigliceridemia y bajas concentraciones de HDL, incrementando de forma gradual la dosis para disminuir la sintomatología gastrointestinal. Es muy importante promover el consumo frecuente de fuentes naturales de ácidos grasos poliinsaturados en la dieta habitual.

En la Figura 2 se mencionan las principales implicaciones clínicas e inmunológicas de los micronutrientes más estudiados en los individuos sanos, así como en población VIH+.

\section{Otros micronutrientes}

Además de los nutrientes ya mencionados, se sabe que las vitaminas A, B, C y E actúan en el sistema inmunológico como hormonas o co-factores ${ }^{(65)}$.

La deficiencia de vitamina A se ha asociado a mayor morbi-mortalidad en individuos infectados con el $\mathrm{VIH}^{(66)}$. Se han realizado estudios para evaluar su rol en la progresión a SIDA, pero los resultados no han sido concluyentes. La mayoría de los estudios de suplementación de vitamina A se han realizado en mujeres embarazadas con el objetivo de evaluar la transmisión vertical del VIH, sin mostrar resultados positivos según las observaciones de un metaanálisis publicado en el $2017^{(67)}$. En población adulta con el VIH y en población coinfectada con tuberculosis, la suplementación de $5.000 \mathrm{UI} /$ día de vitamina A durante un mes no disminuyó parámetros de estrés oxidativo ${ }^{(68)}$. 
A Beneficios potenciales asociados al consumo de micronutrientes en diferentes condiciones, en ausencia de infección por el VIH

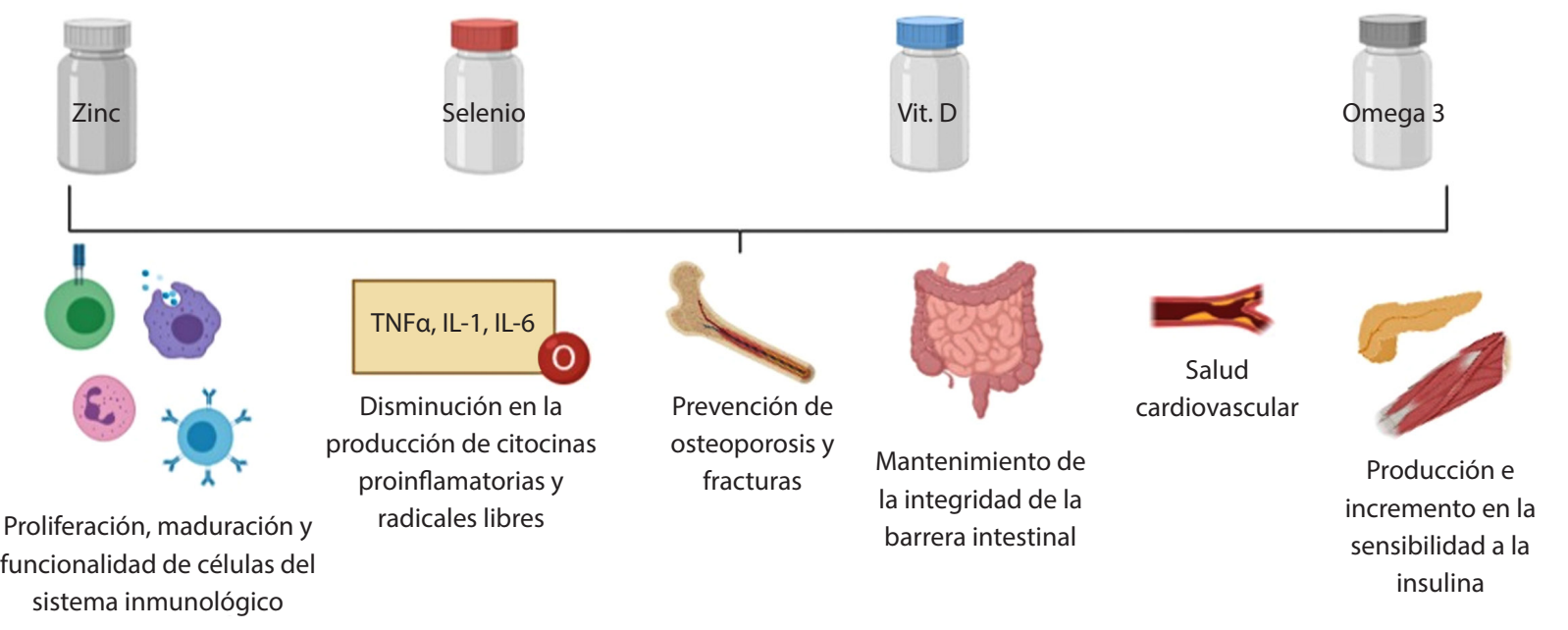

B Efectos observados en estudios de suplementación con micronutrientes en individuos infectados con el VIH
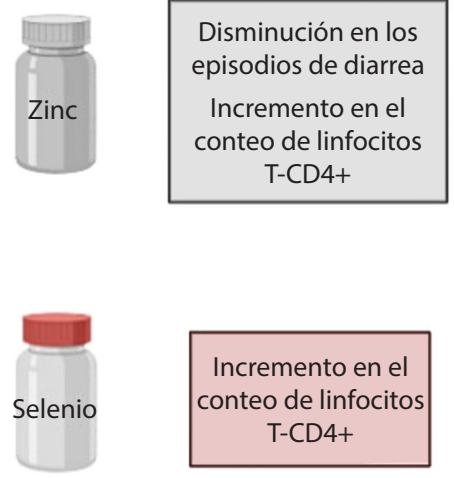

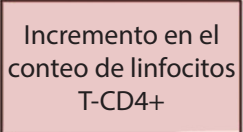

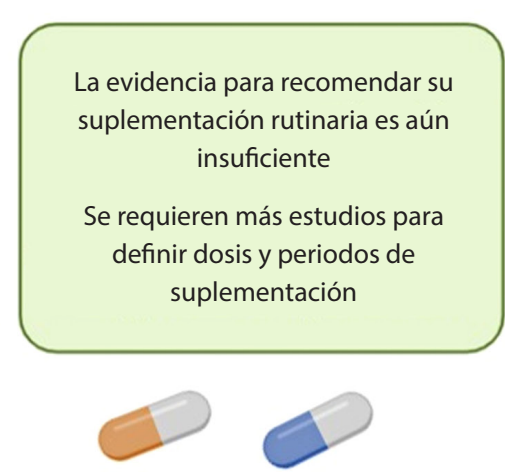

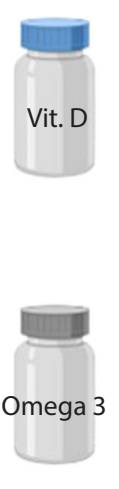

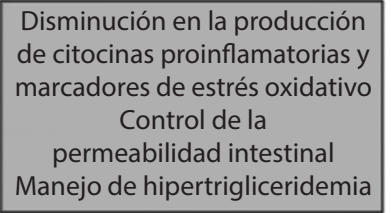

Figura 2. Efecto clínico e inmunológico de los micronutrientes en individuos VIH negativos e infectados con el VIH. Se ha estudiado el efecto clínico e inmunológico de la suplementación de zinc, selenio, vitamina D y omega 3 en individuos con y sin infección por el VIH. A. En individuos sin infección por el VIH estos micronutrientes han mostrado tener efecto sobre la proliferación, maduración y funcionalidad del sistema inmunológico, además de reducir las concentraciones de citocinas proinflamatorias y marcadores de estrés oxidativo. B. En individuos VIH+ existe evidencia de posibles beneficios sobre diversos parámetros clínicos e inmunológicos; sin embargo, la evidencia es insuficiente. Diseñada por los autores.

Las vitaminas del complejo B tienen un rol importante en la inmunidad adaptativa, en la maduración de linfocitos T y la funcionalidad de neutrófilos en condiciones fisiológicas. Se ha documentado una asociación entre deficiencia sérica de niacina, B12, B1, B2 y B6 con progresión acelerada de la infección ${ }^{(69)}$; sin embargo, no se cuenta con ensayos clínicos que hayan evaluado el efecto de la suplementación de vitaminas del complejo $\mathrm{B}$ en pacientes $\mathrm{VIH}+$.

Algunos estudios han evaluado el impacto de la suplementación de cocteles de vitaminas (multivitamí- nicos) en individuos VIH+. Un metaanálisis publicado por Carter et al. en 2015 reportó una reducción en la progresión de la enfermedad y menor mortalidad en pacientes con VIH sin TAR tras la suplementación ${ }^{(70)}$. Otro metaanálisis publicado en 2017 evaluó el efecto de la suplementación con multivitamínicos en dosis estándar y dosis elevadas, no observándose efectos positivos en mortalidad, cuenta de linfocitos T-CD4+ en sangre periférica y la carga viral ${ }^{(71)}$.

Existen pocos estudios clínicos realizados en pacientes VIH+ con TAR en control virológico que evalúen la 
suplementación con múltiples micronutrientes. Si bien los metaanálisis de estudios que dan suplementación no han mostrado tener un impacto importante en el estado inmunológico, se debe valorar la suplementación en aquellos casos en los que se diagnostique consumo subóptimo o deficiencia sérica de uno o varios nutrientes con el fin de repletar las reservas corporales y evitar las consecuencias de la deficiencia. Se deben evitar aquellos productos comerciales que contienen compuestos herbolarios ante las posibles interacciones con el metabolismo de muchos fármacos, particularmente los antirretrovirales.

\section{RECOMENDACIONES}

No existe consenso respecto a los nutrientes más importantes o las dosis óptimas para suplementar a individuos que viven con el VIH. Así mismo, no existe un algoritmo definido para el diagnóstico de estados de deficiencia de micronutrientes en esta población. La pauta para identificar posibles estados de deficiencia es la evaluación del estado nutricional exhaustiva en cada paciente $\mathrm{VIH}+$, utilizando indicadores del $\mathrm{ABCD}$; antropometría (retraso en el crecimiento, composición corporal), bioquímicos (biometría hemática, perfil de lípidos, glucosa sérica, proteína $\mathrm{C}$ reactiva, nutrientes en sangre), clínicos (examen físico orientado a la nutrición para identificar signos de deficiencias de nutrientes en piel, uñas y cabello) y dietéticos (cuantificación de consumo de nutrientes clave a través de recordatorio de 24 horas o diarios de alimentos). Dichos hallazgos son la base para diseñar la terapia nutricional y la estrategia de suplementación más adecuada para cada individuo. En la Figura 3 se mencionan algunos aspectos básicos a considerar en la práctica clínica para el diagnóstico de deficiencia de micronutrientes en individuos VIH+.

Si bien los resultados observados en los estudios de suplementación de individuos con el VIH muestran resultados que pueden ser contradictorios, esto no justifica que no se brinde una intervención en los casos en los que se identifique un consumo subóptimo o deficiencia sérica. Dicha intervención tendría como objetivo normalizar el estado nutricional del individuo, evitando las consecuencias de estados prolongados de deficiencia.

El campo de investigación de la inmunonutrición es un área científica en crecimiento que busca entender, desde una perspectiva científica, los beneficios medibles de una suplementación. Hacen falta más estudios

\begin{tabular}{|c|}
\hline $\begin{array}{l}\text { Evaluar el estado nutricional } \\
\text { - Antropometría (retraso en el crecimiento, composición corporal). } \\
\text { - Bioquímicos (biometría hemática, perfil de lípidos, glucosa sérica, proteína c reactiva, nutrientes en sangre). } \\
\text { - Clínicos (identificación de signos de deficiencia nutricional en uñas, piel y cabello). } \\
\text { - Dietéticos (evaluación de consumo de nutrientes clave a través de recordatorio de } 24 \text { horas o diarios de alimentos). }\end{array}$ \\
\hline$\downarrow$ \\
\hline $\begin{array}{l}\text { En caso de posible deficiencia (concentraciones séricas o signo de deficiencia), consumo subóptimo en dieta o alteración } \\
\text { metabólica (dislipidemia), valorar la suplementación del nutriente identificado. }\end{array}$ \\
\hline$\downarrow$ \\
\hline $\begin{array}{l}\text { Propuesta de suplementación } \\
\text { - Zinc: < } 40 \mathrm{mg} / \text { día en individuos con TAR en control virológico que no han mostrado reconstitución inmunológica y en } \\
\text { pacientes con diarrea crónica. } \\
\text { - Selenio: sin evidencia de beneficio tras la suplementación. } \\
\text { - Vitamina D: } 4000 \mathrm{UI} / \text { día en individuos con deficiencia sérica hasta llevar las concentraciones a la normalidad (>30 ng/mL). } \\
\text { - Omega 3: } 2 \text { - } 3 \mathrm{~g} / \text { día en individuos con colesterol HDL bajo e hipertrigliceridemia. Iniciar a dosis bajas e incrementar } \\
\text { gradualmente. } \\
\text { - No se recomienda prescribir multivitamínicos de forma generalizada. Evitar productos con compuestos herbolarios. }\end{array}$ \\
\hline$\downarrow$ \\
\hline $\begin{array}{l}\text { Promover el consumo de una dieta saludable, rica en fuentes de zinc, selenio, vitamina D y fuentes de ácidos grasos } \\
\text { poliinsaturados en todos los pacientes. }\end{array}$ \\
\hline
\end{tabular}

Figura 3. Recomendaciones para la atención nutricional del paciente con VIH. Diseñada por los autores. 
clínicos con diseños metodológicos rigurosos que permitan responder a las preguntas de investigación sobre el efecto clínico e inmunológico de la suplementación de micronutrientes en individuos con el VIH.

\section{CONCLUSIÓN}

La suplementación de micronutrientes y multivitamínicos puede ser de utilidad para corregir condiciones de deficiencia, principalmente en quienes tienen un consumo subóptimo o dietas no saludables. Dicha suplementación debería restablecer las reservas del cuerpo y podría tener efectos benéficos para el sistema inmunológico de los individuos. Aunque no existe evidencia sólida que muestre mejoría clínica tras la suplementación en individuos $\mathrm{VIH}+$, es necesario hacer un diagnóstico nutricional exhaustivo de esta población, aunque estos se encuentren clínicamente estables, con carga viral indetectable y sin infecciones oportunistas activas, ya que se considera una población vulnerable por cuestiones inherentes a su padecimiento. Se requieren más ensayos clínicos que incluyan un mayor número de participantes VIH+ en TAR y que evalúen por tiempos más prolongados el impacto de la suplementación en parámetros clínicos e inmunológicos.

\section{Declaración de autoría}

Todos los autores han participado en la concepción y realización del trabajo que ha dado como resultado el artículo en cuestión. I.A.O.P y N.C.R.M. participaron en el análisis de los resultados; AAV participó en la redacción del trabajo, O.B. participó en la redacción y edición del trabajo. Todos los autores revisaron el artículo y validaron su versión final.

\section{Declaración de conflictos de interés}

Sin conflictos de interés por declarar.

\section{Financiamiento}

El presente estudio no recibió financiamiento.

\section{Referencias bibliográficas}

1. Serrão R, Piñero C, Velez J, Coutinho D, Maltez F, Lino S, et al. Non-AIDS-related comorbidities in people living with HIV-1 aged 50 years and older: The AGING POSITIVE study. Int J Infect Dis. 2019; 79: 94-100. doi: https://doi.org/10.1016/j. ijid.2018.10.011.
2. O'Cofaigh E, Lewthwaite P. Natural history of HIV and AIDS. Medicine. 2013;41(8):411-6. doi: https://doi.org/10.1016/j. mpmed.2013.05.009.

3. del Rio C. The global HIV epidemic: What the pathologist needs to know. Semin Diagn Pathol. 2017;34(4):314-7. doi: https://doi.org/10.1053/j.semdp.2017.05.001.

4. Deeks SG, Tracy R, Douek DC. Systemic effects of inflammation on health during chronic HIV infection. Immunity. 2013;39(4):633-45. doi: https://doi.org/10.1016/j. immuni.2013.10.001.

5. Kaplan-Lewis E, Aberg JA, Lee M. Aging with HIV in the ART era. Semin Diagn Pathol. 2017;34(4):384-97. doi: https:// doi.org/10.1053/j.semdp.2017.04.002.

6. Marcus JL, Leyden WA, Alexeeff SE, Anderson AN, Hechter $\mathrm{RC}, \mathrm{Hu} \mathrm{H}$, et al. Comparison of Overall and ComorbidityFree Life Expectancy Between Insured Adults With and Without HIV Infection, 2000-2016. JAMA Netw Open. 2020;3(6):e207954. doi: https://doi.org/10.1001/jamanetworkopen.2020.7954.

7. Mankal PK, Kotler DP. From wasting to obesity, changes in nutritional concerns in HIV/AIDS. Endocrinol Metab Clin North Am. 2014;43(3):647-63. doi: https://doi. org/10.1016/j.ecl.2014.05.004.

8. Funderburg NT, Mehta NN. Lipid Abnormalities and Inflammation in HIV Inflection. Curr HIV/AIDS Rep. 2016;13(4):218-25. doi: https://doi.org/10.1007/s11904016-0321-0.

9. Warriner AH, Burkholder GA, Overton ET. HIV-related metabolic comorbidities in the current ART era. Infect Dis Clin North Am. 2014; 28(3):457-76. doi: https://doi. org/10.1016/j.idc.2014.05.003.

10. Gil L, Tarinas A, Hernández D, Vega RB, Pérez D, Tápanes $\mathrm{R}$, et al. Altered oxidative stress indexes related to disease progression marker in human immunodeficiency virus infected patients with antiretroviral therapy. Biomed Aging Pathol. 2011;1(1):8-15. doi: https://doi.org/10.1016/j.biomag.2010.09.001.

11. Utay NS, Hunt PW. Role of immune activation in progression to AIDS. Curr Opin HIV AIDS. 2016;11(2):131-7. doi: https://doi.org/10.1097/COH.0000000000000242.

12. Akiyama H, Gummuluru S. HIV-1 Persistence and Chronic Induction of Innate Immune Responses in Macrophages. Viruses. 2020;12(7):711. doi: https://doi.org/10.3390/ v12070711.

13. Liang $\mathrm{H}$, Xie Z, Shen T. Monocyte activation and cardiovascular disease in HIV infection. Cell Mol Immunol. 2017;14(12):9602. doi: https://doi.org/10.1038/cmi.2017.109.

14. Longenecker CT, Sullivan C, Baker JV. Immune Activation and Cardiovascular Disease in Chronic HIV Infection. Curr Opin HIV AIDS. 2016;11(2): 216-25. doi: https://doi. org/10.1097/COH.0000000000000227.

15. Goh SSL, Lai PSM, Tan ATB, Ponnampalavanar S. Reduced bone mineral density in human immunodeficiency virus- 
infected individuals: a meta-analysis of its prevalence and risk factors. Osteoporos Int. 2018; 29(3): 595-613. doi: https:// doi.org/10.1007/s00198-017-4305-8.

16. Somsouk M, Estes JD, Deleage C, Dunham RM, Albright R, Inadomi JM, et al. Gut epithelial barrier and systemic inflammation during chronic HIV infection. AIDS. 2015;29(1):4351. doi: https://doi.org/10.1097/QAD.0000000000000511.

17. Assimakopoulos SF, Dimitropoulou D, Marangos M, Gogos CA. Intestinal barrier dysfunction in HIV infection: pathophysiology, clinical implications and potential therapies. Infection. 2014;42(6):951-9. doi: https://doi.org/10.1007/ s15010-014-0666-5.

18. Pedersen KK, Pedersen M, Trøseid M, Gaardbo JC, Lund $\mathrm{TT}$, Thomsen C, et al. Microbial translocation in HIV infection is associated with dyslipidemia, insulin resistance, and risk of myocardial infarction. J Acquir Immune Defic Syndr. 2013;64(5):425-33. doi: https://doi.org/10.1097/ QAI.0b013e31829f919d.

19. Logan C, Beadsworth MBJ, Beeching NJ. HIV and diarrhoea: what is new? Curr Opin Infect Dis. 2016;29(5):486-94. doi: https://doi.org/10.1097/QCO.0000000000000305.

20. de Pee S, Semba RD. Role of nutrition in HIV infection: review of evidence for more effective programming in resource-limited settings. Food Nutr Bull. 2010;31(4):S313-344.

21. Livingstone C. Zinc: physiology, deficiency, and parenteral nutrition. Nutr Clin Pract. 2015;30(3):371-82. doi: https:// doi.org/10.1177/0884533615570376.

22. Bonaventura P, Benedetti G, Albarède F, Miossec P. Zinc and its role in immunity and inflammation. Autoimmun Rev. 2015;14(4):277-85. doi: https://doi.org/10.1016/j. autrev.2014.11.008.

23. Martinez SS, Campa A, Li Y, Fleetwood C, Stewart T, Ramamoorthy V, et al. Low Plasma Zinc Is Associated with Higher Mitochondrial Oxidative Stress and Faster Liver Fibrosis Development in the Miami Adult Studies in HIV Cohort. J Nutr. 2017;147(4):556-62. doi: https://doi. org/10.3945/jn.116.243832.

24. Poudel KC, Bertone-Johnson ER, Poudel-Tandukar K. Serum Zinc Concentration and C-Reactive Protein in Individuals with Human Immunodeficiency Virus Infection: the Positive Living with HIV (POLH) Study. Biol Trace Elem Res. 2016;171(1):63-70. doi: https://doi.org/10.1007/s12011015-0520-3.

25. Osuna-Padilla IA, Briceño O, Aguilar-Vargas A, RodríguezMoguel NC, Villazon-De la Rosa A, Pinto-Cardoso S, et al. Zinc and selenium indicators and their relation to immunologic and metabolic parameters in male patients with human immunodeficiency virus. Nutrition. 2020;70:110585. doi: https://doi.org/10.1016/j.nut.2019.110585.

26. Mocchegiani E, Veccia S, Ancarani F, Scalise G, Fabris N. Benefit of oral zinc supplementation as an adjunct to zidovudine (AZT) therapy against opportunistic infections in AIDS. Int J Immunopharmacol. 1995;17(9):719-27. doi: https:// doi.org/10.1016/0192-0561(95)00060-F.
27. Bobat R, Coovadia H, Stephen C, Naidoo KL, McKerrow $\mathrm{N}$, Black RE, et al. Safety and efficacy of zinc supplementation for children with HIV-1 infection in South Africa: a randomised double-blind placebo-controlled trial. Lancet. 2005;366(9500):1862-7. doi: https://doi.org/10.1016/ S0140-6736(05)67756-2.

28. Baum MK, Lai S, Sales S, Page JB, Campa A. Randomized, controlled clinical trial of zinc supplementation to prevent immunological failure in HIV-infected adults. Clin Infect Dis. 2010;50(12):1653-60. doi: https://doi. org/10.1086/652864.

29. Green JA, Lewin SR, Wightman F, Lee M, Ravindran TS, Paton NI. A randomised controlled trial of oral zinc on the immune response to tuberculosis in HIV-infected patients. Int J Tuberc Lung Dis. 2005;9(12):1378-84.

30. Cárcamo C, Hooton T, Weiss NS, Gilman R, Wener MH, Chavez V, et al. Randomized controlled trial of zinc supplementation for persistent diarrhea in adults with HIV-1 infection. J Acquir Immune Defic Syndr. 2006;43(2):197-201. doi: https://doi.org/10.1097/01.qai.0000242446.44285.b5.

31. Fawzi WW, Villamor E, Msamanga GI, Antelman G, Aboud $\mathrm{S}$, Urassa W, et al. Trial of zinc supplements in relation to pregnancy outcomes, hematologic indicators, and $\mathrm{T}$ cell counts among HIV-1-infected women in Tanzania. Am J Clin Nutr. 2005;81(1):161-7. doi: https://doi.org/10.1093/ ajcn/81.1.161.

32. Villamor E, Aboud S, Koulinska IN, Kupka R, Urassa W, Chaplin B, et al. Zinc supplementation to HIV-1-infected pregnant women: effects on maternal anthropometry, viral load, and early mother-to-child transmission. Eur J Clin Nutr. 2006;60(7):862-9. doi: https://doi.org/10.1038/ sj.ejcn. 1602391 .

33. Institute of Medicine (US) Panel on Micronutrients. Dietary Reference Intakes for Vitamin A, Vitamin K, Arsenic, Boron, Chromium, Copper, Iodine, Iron, Manganese, Molybdenum, Nickel, Silicon, Vanadium, and Zinc. Washington (DC): National Academies Press (US); 2001.

34. Avery JC, Hoffmann PR. Selenium, Selenoproteins, and Immunity. Nutrients. 2018;10(9):1203. doi: https://doi. org/10.3390/nu10091203.

35. Brigelius-Flohé R, Flohé L. Selenium and redox signaling. Arch Biochem Biophys. 2017;617:48-59. doi: https://doi. org/10.1016/j.abb.2016.08.003.

36. Pugliese C, Patin RV, Palchetti CZ, Chiantelli CC, Thomé BGAF, de Menezes SRC, et al. Assessment of antioxidants status and superoxide dismutase activity in HIV-infected children. Braz J Infect Dis. 2014;18(5):481-6. doi: https://doi. org/10.1016/j.bjid.2014.02.003.

37. Shivakoti R, Christian P, Yang W-T, Gupte N, Mwelase N, Kanyama $\mathrm{C}$, et al. Prevalence and risk factors of micronutrient deficiencies pre- and post-antiretroviral therapy (ART) among a diverse multicountry cohort of HIV-infected adults. Clin Nutr. 2016;35(1):183-9. doi: https://doi.org/10.1016/j. clnu.2015.02.002. 
38. Amare TB, Tafess TK, Ota F, Moges F, Moges B, Andualem B, et al. Serum Concentration of Selenium in Diarrheic Patients with and without HIV/AIDS in Gondar, Northwest Ethiopia. J AIDS Clin Res. 2011;2:6. doi: https://doi. org/10.4172/2155-6113.1000128.

39. Hurwitz BE, Klaus JR, Llabre MM, Gonzalez A, Lawrence PJ, Maher KJ, et al. Suppression of human immunodeficiency virus type 1 viral load with selenium supplementation: a randomized controlled trial. Arch Intern Med. 2007;167(2):14854. doi: https://doi.org/10.1001/archinte.167.2.148.

40. Kamwesiga J, Mutabazi V, Kayumba J, Tayari JCK, Uwimbabazi JC, Batanage G, et al. Effect of selenium supplementation on CD4+ T-cell recovery, viral suppression and morbidity of HIV-infected patients in Rwanda: a randomized controlled trial. AIDS. 2015;29(9):1045-52. doi: https://doi. org/10.1097/QAD.0000000000000673.

41. Hadadi A, Ostovar A, Noor BE, Rasoolinejad M, Abdolbaghi $\mathrm{MH}$, Yousefi $\mathrm{S}$, et al. The effect of selenium and zinc on CD4(+) count and opportunistic infections in HIV/AIDS patients: a randomized double blind trial. Acta Clin Belg. 2020;75(3):170-6. doi: https://doi.org/10.1080/17843286 .2019 .1590023 .

42. Institute of Medicine (US) Panel on Dietary Antioxidants and Related Compounds. Dietary Reference Intakes for Vitamin C, Vitamin E, Selenium, and Carotenoids. Washington (DC): National Academies Press (US); 2000.

43. Sassi F, Tamone C, D’Amelio P. Vitamin D: Nutrient, Hormone, and Immunomodulator. Nutrients. 2018;10(11). doi: https://doi.org/10.3390/nu10111656.

44. Jiménez-Sousa MÁ, Martínez I, Medrano LM, Fernández-Rodríguez A, Resino S. Vitamin D in Human Immunodeficiency Virus Infection: Influence on Immunity and Disease. Front Immunol. 2018;9:458. doi: https://doi. org/10.3389/fimmu.2018.00458.

45. Mansueto P, Seidita A, Vitale G, Gangemi S, Iaria C, Cascio A. Vitamin D Deficiency in HIV Infection: Not Only a Bone Disorder. Biomed Res Int. 2015;2015:735615. doi: https:// doi.org/10.1155/2015/735615.

46. Akimbekov NS, Ortoski RA, Razzaque MS. Effects of sunlight exposure and vitamin D supplementation on HIV patients. J Steroid Biochem. 2020;200:105664. doi: https://doi. org/10.1016/j.jsbmb.2020.105664.

47. Manion M, Hullsiek KH, Wilson EMP, Rhame F, Kojic E, Gibson D, et al. Vitamin D deficiency is associated with IL-6 levels and monocyte activation in HIV-infected persons. PLoS One. 2017;12(5):e0175517. doi: https://doi.org/10.1371/ journal.pone.0175517.

48. Calza L, Borderi M, Granozzi B, Malosso P, Pancaldi L, Bon I, et al. Vitamin D insufficiency is associated with subclinical atherosclerosis in HIV-1-infected patients on combination antiretroviral therapy. HIV Research \& Clinical Practice. 2019;20(6):131-9. doi: https://doi.org/10.1080/25787489 .2020 .1724749 .
49. Eckard AR, O’Riordan MA, Rosebush JC, Lee ST, Habib JG, Ruff JH, et al. Vitamin D supplementation decreases immune activation and exhaustion in HIV-1-infected youth. Antivir Ther. 2018; 23(4):315-24. doi: https://doi.org/10.3851/ IMP3199.

50. Eckard AR, O'Riordan MA, Rosebush JC,RuffJH, Chahroudi A, Labbato D, et al. Effects of Vitamin D Supplementation on Bone Mineral Density and Bone Markers in HIV-Infected Youth. J Acquir Immune Defic Syndr. 2017;76(5): 539-46. doi: https://doi.org/10.1097/QAI.0000000000001545.

51. Havens PL, Stephensen CB, Van Loan MD, Schuster GU, Woodhouse LR, Flynn PM, et al. Vitamin D3 Supplementation Increases Spine Bone Mineral Density in Adolescents and Young Adults With Human Immunodeficiency Virus Infection Being Treated With Tenofovir Disoproxil Fumarate: A Randomized, Placebo-Controlled Trial. Clin Infect Dis. 2018;66(2):220-8. doi: https://doi.org/10.1093/cid/cix753.

52. Yin MT, RoyChoudhury A, Bucovsky M, Colon I, Ferris DC, Olender S, et al. A Randomized Placebo-Controlled Trial of Low- Versus Moderate-Dose Vitamin D3 Supplementation on Bone Mineral Density in Postmenopausal Women With HIV. J Acquir Immune Defic Syndr. 2019; 80(3):342-9. doi: https://doi.org/10.1097/QAI.0000000000001929.

53. Institute of Medicine (US) Committee to Review Dietary Reference Intakes for Vitamin D and Calcium. Dietary Reference Intakes for Calcium and Vitamin D. Washington (DC): National Academies Press (US); 2011.

54. Radzikowska U, Rinaldi AO, Çelebi SZ, Karaguzel D, Wojcik $\mathrm{M}$, Cypryk K, et al. The Influence of Dietary Fatty Acids on Immune Responses. Nutrients. 2019;11(12):2990. doi: https://doi.org/10.3390/nu11122990.

55. Metkus TS, Timpone J, Leaf D, Bidwell Goetz M, Harris WS, Brown TT. Omega-3 fatty acid therapy reduces triglycerides and interleukin-6 in hypertriglyeridemic HIV patients. HIV Med. 2013;14(9):530-9. doi: https://doi.org/10.1111/ hiv.12046.

56. Coghill AE, SchenkJM, Mahkoul Z, Orem J, Phipps W, Casper C. Omega-3 decreases IL-6 levels in HIV and human HHV-8 co-infected patients: results from a randomized supplementation trial in Uganda. AIDS. 2018;32(4):505-12. doi: https:// doi.org/10.1097/QAD.0000000000001722.

57. Amador-Licona N, Díaz-Murillo TA, Gabriel-Ortiz G, Pacheco-Moises FP, Pereyra-Nobara TA, Guízar-Mendoza JM, et al. Omega 3 Fatty Acids Supplementation and Oxidative Stress in HIV-Seropositive Patients. A Clinical Trial. PLoS One. 2016;11(3). doi: https://doi.org/10.1371/ journal.pone.0151637.

58. Zhang Y-G, Xia Y, Lu R, Sun J. Inflammation and intestinal leakiness in older HIV+ individuals with fish oil treatment. Genes Dis. 2018;5(3):220-5. doi: https://doi.org/10.1016/j. gendis.2018.07.001.

59. Peters BS, Wierzbicki AS, Moyle G, Nair D, Brockmeyer N. The effect of a 12-week course of omega-3 polyunsaturated 
fatty acids on lipid parameters in hypertriglyceridemic adult HIV-infected patients undergoing HAART: a randomized, placebo-controlled pilot trial. Clin Ther. 2012;34(1):67-76. doi: https://doi.org/10.1016/j.clinthera.2011.12.001.

60. Oliveira JM, Rondó PHC, Yudkin JS, Souza JMP, Pereira TN, Catalani AW, et al. Effects of fish oil on lipid profile and other metabolic outcomes in HIV-infected patients on antiretroviral therapy: a randomized placebo-controlled trial. Int J STD AIDS. 2014;25(2):96-104. doi: https://doi. org/10.1177/0956462413513748.

61. Domingo P, Gallego-Escuredo JM, Fernández I, Villarroya J, Torres F, Gutierrez MDM, et al. Effects of docosahexanoic acid supplementation on inflammatory and subcutaneous adipose tissue gene expression in HIV-infected patients on combination antiretroviral therapy (cART). A sub-study of a randomized, double-blind, placebo-controlled study. Cytokine. 2018;105:73-9. doi: https://doi.org/10.1016/j. cyto.2018.02.008.

62. Domingo P, Fernández I, Gallego-Escuredo JM, Torres F, Gutierrez MDM, Mateo MG, et al. Effects of docosahexanoic acid on metabolic and fat parameters in HIV-infected patients on cART: A randomized, double-blind, placebo-controlled study. Clin Nutr. 2018;37(4):1340-7. doi: https://doi. org/10.1016/j.clnu.2017.05.032.

63. Oliveira JM, Rondó PHC. Omega-3 fatty acids and hypertriglyceridemia in HIV-infected subjects on antiretroviral therapy: systematic review and meta-analysis. HIV Clin Trials. 2011;12(5):268-74. doi: https://doi.org/10.1310/hct1205268.

64. Fogacci F, Strocchi E, Veronesi M, Borghi C, Cicero AFG. Effect of Omega-3 Polyunsaturated Fatty Acids Treatment on Lipid Pattern of HIV Patients: A Meta-Analysis of
Randomized Clinical Trials. Mar Drugs. 2020;18(6):292. doi: https://doi.org/10.3390/md18060292.

65. Shah KK, Verma R, Oleske JM, Scolpino A, Bogden JD. Essential trace elements and progression and management of HIV infection. Nutr Res. 2019;71:21-9. doi: https://doi. org/10.1016/j.nutres.2019.08.001

66. Mehta S, Fawzi W. Effects of vitamins, including vitamin A, on HIV/AIDS patients. Vitam Horm. 2007;75:355-83. doi: https://doi.org/10.1016/S0083-6729(06)75013-0.

67. Wiysonge CS, Ndze VN, Kongnyuy EJ, Shey MS, Cochrane Infectious Diseases Group. Vitamin A supplements for reducing mother-to-child HIV transmission. Cochrane Database Syst Rev. 2017;(9): CD003648. doi: https://doi. org/10.1002/14651858.CD003648.pub4.

68. Makinde O, Rotimi K, Ikumawoyi V, Adeyemo T, Olayemi S. Effect of vitamin A and vitamin $\mathrm{C}$ supplementation on oxidative stress in HIV and HIV-TB co-infection at Lagos University Teaching Hospital (LUTH) Nigeria. Afr Health Sci. 2017;17(2):308-14. doi: https://doi.org/10.4314/ahs. v17i2.3.

69. Drain PK, Kupka R, Mugusi F, Fawzi WW. Micronutrients in HIV-positive persons receiving highly active antiretroviral therapy. Am J Clin Nutr. 2007;85(2):333-45. doi: https:// doi.org/10.1093/ajen/85.2.333.

70. Carter GM, Indyk D, Johnson M, Andreae M, Suslov K, Busani S, et al. Micronutrients in HIV: a Bayesian metaanalysis. PLoS One. 2015;10(4):e0120113. doi: https://doi. org/10.1371/journal.pone.0120113.

71. Visser ME, Durao S, Sinclair D, Irlam JH, Siegfried N. Micronutrient supplementation in adults with HIV infection. Cochrane Database Syst Rev. 2017;5(5): CD003650. doi: https://doi.org/10.1002/14651858.CD003650.pub4. 